\title{
THE TREATMENT OF PULMONARY TUBERCULOSIS BY SURGICAL RESECTION
}

\author{
By Walter L. Phillips, M.R.C.P.(Lond.), F.R.C.S.(Eng.). \\ Thoracic Surgeon, Groote Schuur Hospital; Thoracic Surgeon, City Hospital for Infectious Diseases, Cape Town
}

Many years have passed since surgical treatment for cases of pulmonary tuberculosis was first instituted, and with this lapse of time the simple surgical measures first employed have been supplemented by major thoracic procedures. Recently major surgical operations such as pneumonectomy and lobectomy have been performed. The successful advent of these procedures in the field of therapy for pulmonary tuberculosis has largely been due to the improvement in surgical technique, the introduction of special anaesthesia and the use of specific antibiotics.

Pulmonary resection has for many years been a standard operation. Its place in the treatment of cases of tuberculosis resulted from the fact that when pulmonary lobes which had been removed for bronchiectasis were histologically examined, a coincident tuberculosis was often discovered, and yet in these cases the incision generally healed by first intention and the post-operative course was uneventful. Removal of affected or diseased tissue is a surgical axiom, and thus deliberate attempts were made to resect the tuberculous lung tissue. Many disastrous sequelae, however, resulted in the abandoning of resection as a line of treatment, until the more recent advances in anaesthesia and the newer antibiotics reduced the hazards of this method.

A very different picture is seen today. This paper gives a report on $5^{\circ}$ cases of pulmonary tuberculosis treated by surgical resection. Pneumonectomy and lobectomy are discussed, as well as segmental resection, which refers to the removal of an anatomical segment of a lobe. Pleurectomy is also described, as the pleural membranes are often involved in the tuberculous process or are affected by ineffective attempts at treatment. In many cases part of the pleural lining has been resected, and in some cases removal of the entire pleural covering has been necessary.

\section{Types of Tuberculous Pulmonary Disease}

The indications for surgical resection should be clearly outlined. Unfortunately the surgeon is usually only asked to treat the patient when other measures have failed, and the treatment is far too sharply divided into medical and surgical stages.
Many accepted views and lines of treatment require to be re-assessed, and many of the older medical and surgical methods will have to be permanently discarded. It is as important to know when medical treatment should be supplanted by surgery as it is to realize when surgical therapy is primarily indicated.

We shall consider pulmonary tuberculosis under two headings in order to elaborate on this theme.

\section{The Temporary Type of Disease}

This group includes those cases in which the pulmonary lesion heals, leaving only a small calcified or fibrosed area with relatively unimpaired respiratory function. Bed rest, antibiotic treatment, pneumothorax and pneumoperitoneum therapy are the methods usually adopted. Occasionally minor surgical operations such as thoracoscopy and adhesion section, and phrenic paralysis are used in conjunction with the above.

Thus the patient is rendered non-infectious, the pulmonary function is restored and at no stage has he been exposed to dangerous complications. At.the termination of treatment careful medical and radiological observation should be maintained.

\section{The Permanent Type of Disease}

These cases show permanent damage to the entire lung or portion of $i t$, and restoration of normal function is not possible. Any temporary form of treatment, even though it may render the patient non-infectious, will leave him with impaired pulmonary function and with an increased susceptibility to dangerous sequelae. Generally at this stage the patients were referred for surgical treatment and the operation of thoracoplasty and apicolysis was performed. It was recognized that where permanent damage to the lung had occurred a permanent form of collapse therapy was indicated.

Resection is advocated as an extension of this principle, and if the patient's general condition is satisfactory the permanently damaged, useless and dangerous lung tissue should be removed. It is obvious that the surgeon should collaborate with the physician at the primary institution of treatment. 


\section{Indications for Pulmonary Resection}

The lung can be divided into a number of anatomical segments, each segment having its own bronchus, artery and vein. This implies that any one segment of any anatomical lobe of either lung should be regarded as an anatomical entity, which can be removed from the lobe through a plane of cleavage which is avascular and free of bronchial tributaries.

\section{Extensive Unilateral and Unilobar Tuberculosis}

It has been previously stated that resection is indicated in any case showing permanent disorganization of a lobe or of the entire lung. In the past, artificial pneumothorax was employed as the line of treatment for these patients and a good pneumothorax was often obtained, though an adhesion section was sometimes necessary.

The pneumothorax in extensively diseased cases invariably led to an atelectasis of the affected part. Examination of sputum was usually negative for tubercle bacilli, but the patient was deemed fortunate if he escaped any complications such as pleurisy or empyema. After three to five years of this treatment the pneumothorax was usually abandoned, and with the aeration of the lung the cavities reappeared and the sputum became positive again. Aeration was often incomplete, gross mediastinal displacement was seen and very soon symptoms of bronchiectasis of the affected lung and emphysema of the other lung were superimposed on the already gloomy picture.

Some patients refused to give up artificial pneumothorax therapy as they had felt -so well during the course.

Frequently a pneumothorax was not obtainable and a thoracoplasty was performed. This major procedure not only carries with it a mortality rate a little higher than that for a lobectomy or pneumonectomy, but the diseased tissue which is retained in the chest remains as a possible site for the development of more advanced pathology.

\section{Pulmonary and Endobronchial Tuberculosis}

Endobronchial tuberculosis with early, reversible, pulmonary parenchymal disease is an absolute contraindication to collapse therapy. This view applies even more strongly to cases of advanced disease. Complete obstruction of the bronchus, with the complication of lung abscess formation, may follow on the induction of an artificial pneumothorax.

Endobronchitis of the main bronchus is usually associated with disorganization of the lung, and this combination provides a clear indication for pneumonectomy.

Where a tuberculous ulcer in the main bronchus is associated with disease of one lobe and the other lobes appear normal, radiographically, an attempt should first be made to treat the local lesion in the bronchus. It is always important to conserve healthy lung tissue.

Lobectomy is indicated if the bronchial lesion affects only one lobe and if that lobe is partly or wholly involved by the tuberculous process.

Pre-operative bronchoscopy to determine the presence of tuberculous endobronchitis is extremely important. The resection of a lobe involves division of the bronchus, and if this division occurs across an ulcerated site there will be contamination of the pleural cavity.

Pre-operative bronchoscopy has been adopted as a routine measure by our thoracic surgical unit.

A word should be said about the case of tuberculosis with a positive sputum which presents with a main bronchial stenosis. The stenosis is normally not complete and the lung appears to have normal function with no radiographic evidence of disease. The patient should be closely observed and the usual antibiotics should be administered. A pneumonectomy should be performed if any sign of pulmonary involvement ensues.

\section{Failed Pneumothorax Collapse Therapy}

Pneumothorax collapse therapy may fail completely owing to the presence of widespread adhesions attaching the lung to the chest wall, and thus resection of the affected lung tissue becomes the treatment of choice.

In many cases, however, a pneumothorax may be obtained, but dense extensive indivisible adhesions remain attaching the lung to the thoracic cage. If an unclosed cavity still exists the pneumothorax should be immediately abandoned and resection should be carried out. The maintenance of such a pneumothorax invites disaster, as the adhesions may be torn off the lung over the cavity area with inevitable infection of the pleural space, in the shape of an obliterative pleurisy or an empyema.

Occasionally after a pneumothorax induction all the cavities appear to be closed, but there is persistence of large, indivisible adhesions. These cases are similarly potentially dangerous.

A pneumothorax should not be maintained in cases irrespective of the presence of adhesions if an underlying cavity commences to enlarge, as the distension will eventually lead to bursting of the cavity.

A patient may continue to produce a positive sputum even though the pneumothorax is satisfactory, and the disease appears to be under control. Bronchoscopy must be supplemented by bronchography before the decision can be reached that all is well in the collapsed lobe. In one of the cases under review, bronchography revealed the 
presence of a large cavity in a lobe which was believed to be satisfactorily collapsed.

Artificial pneumothorax should only be employed as a means of treatment if it can be adequately performed and if the indications for it are exact. It has been our experience that too many inefficient pneumothoraces have been maintained. Unfortunately far too often the unsatisfactory pneumothorax has been abandoned to be replaced by an equally useless pneumoperitoneum with phrenic paralysis.

\section{Failed Thoracoplasty}

Each year witnesses the return of a number of thoracoplasty cases owing to the presence of a persistently positive sputum. Some cases have a residual cavity easily discernible on ordinary films, though usually special radiographic examinations are essential. Tomography is helpful in many cases but may not entirely exclude the presence of cavitation.

Bronchography has proved extremely important, as in many cases it has demonstrated the presence of bronchiectasis in parts of the lung which had been previously regarded as normal. This bronchiectasis apparently develops after the thoracoplasty operation.

There are many reasons why thoracoplasty may not be considered the operation of choice. If residual cavitation and bronchiectasis persist after thoracoplasty the resection which may then be indicated becomes a more difficult procedure. Many authors have reported a high rate of sputum. conversions and cavity closures following on thoracoplasty operations. Their results vary from 60 to 80 per cent. of successes. In my experience of a large number of thoracoplasty operations with apicolysis, although every attempt has been made to conform to the techniques described by the authors, the percentage of conversions has been under 60 per cent. Apart from the risks of the actual operation the complications are many, and hospitalization and rehabilitation is of long duration.

In our thoracic unit the operation of thoracoplasty is employed to close the spaces which may remain after lung resection, in order to prevent overdistension emphysema. Resection has replaced thoracoplasty in the surgical therapy of pulmonary tuberculosis.

Previously, while the European patient accepted the rationale of the thoracoplasty operation, the Coloured and African patients remained recalcitrant and could not be persuaded to undergo this procedure. Pulmonary resection, which is designed to remove the tuberculous lung tissue and involves a shorter convalescence, is gladly accepted by both European and non-European patients.

\section{Lower Lobe Disease}

Lower lobe disease has, in the past, shown a $\vec{\Rightarrow}$ poor response to treatment, possibly due to the activity which occurs continuously in this area. The lower lobes are never in a state of complete $\frac{\bar{\sigma}}{\bar{D}}$ rest as they are affected by the movements of the $\frac{\rho}{\sigma}$ diaphragm or by the transmitted movements of the $\stackrel{\mathbb{Q}}{\Omega}$ heart.

Pneumothorax and pneumoperitoneum treat- $\overrightarrow{0}$ ment usually fails in cases of lower lobe disease. In most of these patients the disease exists in the $\vec{\omega}$ apex of the lower lobe or the so-called 'superior $\frac{\sigma}{\partial}$ segment' described by American authors, and 0 when cavitation is present it appears radiographic- 3 . ally to be in the region of the pulmonary hilum. Ty Cavitation in the apex of the lower lobe is generally $\omega$ associated with dense, indivisible adhesions between the lung and the paravertebral sulcus, so it $\frac{N}{\sigma}$ is not surprising that a pneumothorax is contraindicated.

\section{Tuberculoma of the Lung}

Tuberculoma of the lung may not be diagnosed prior to operation, but if a history of tuberculos? o has been obtained the nature of a rounded pu․ monary tumour should be suspected. The tuberculomata are often well encapsulated, and many cases the radiographic appearance is that of a tuberculous abscess containing a central mass of necrotic tissue. This lesion may be localized $\stackrel{\varnothing}{\AA}$ enough to permit of a segmental resection of lung $\overrightarrow{\overrightarrow{0}}$ tissue.

\section{Bronchopleural Fistula}

The presence of a bronchopleural fistula in pulmonary tuberculosis usually signifies irreparable lung damage, associated with a large tuberculous $\frac{\hat{\sigma}}{3}$ empyema and great thickening of the pleural lining. The general condition of the patient is invariably serious and the prognosis is grave.

If the bronchopleural fistula is small, some im- 음 provement may be obtained by external under- $\rightarrow$ water drainage of the empyema. If the general condition of the patient can be improved it may N be possible to resect the lung as well as the entire pleural membrane. This procedure, which has $\mathcal{N}^{\circ}$ been described by Sarot, includes not only a $\mathbb{\omega}$ pneumonectomy but also removal of the entire ${ }^{\circ}$ parietal pleura, and is considered to be of value by the author. Haemorrhage is usually quite con- $\mathbb{\Phi}$ siderable and adequate quantities of blood should ? be available for transfusion during the operation.

\section{Resection of the Pleura}

At the termination of artificial pneumothorax

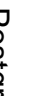




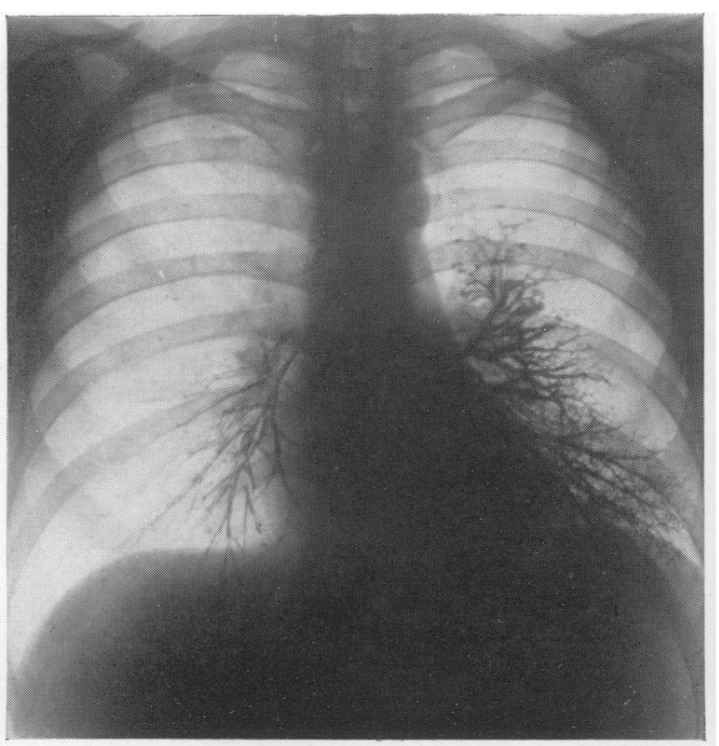

FIg. I.-Normal bronchial distribution in left lung at end of left artificial pneumothorax treatment. Conclusion is that treatment can be abandoned without fear of complications as lung expands.

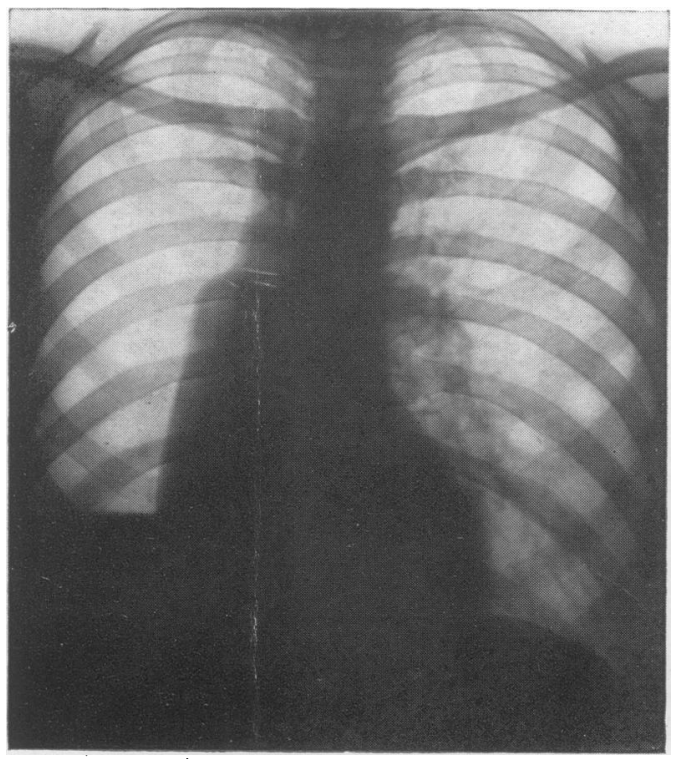

$\mathrm{FI}_{\mathrm{G} .}$ 3.-Complete atelectasis of right lung following artificial pneumothorax for extensive infiltration and cavitation. No sputum. Large pleural effusion containing tubercle bacilli. Right lung permanently disorganized and dangerous complications followed this treatment,

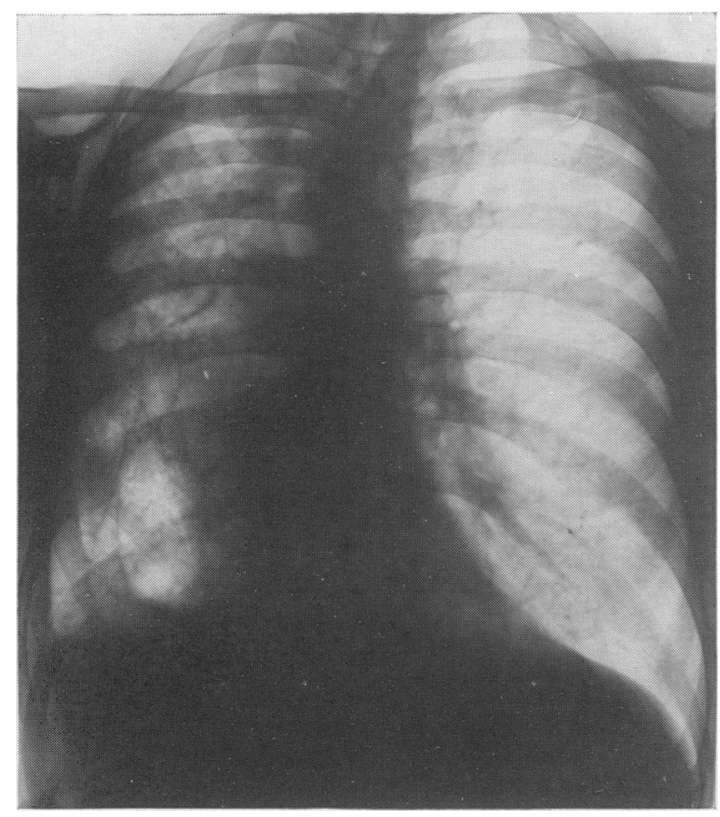

FIG. 2.-Extensive tuberculous disease of right lung with tracheal displacement to right. Right artificial pneumothorax obtained-surprisingly. This method of treatment definitely contraindicated in such case, as right lung permanently disorganized and dangerous complications are inevitable.

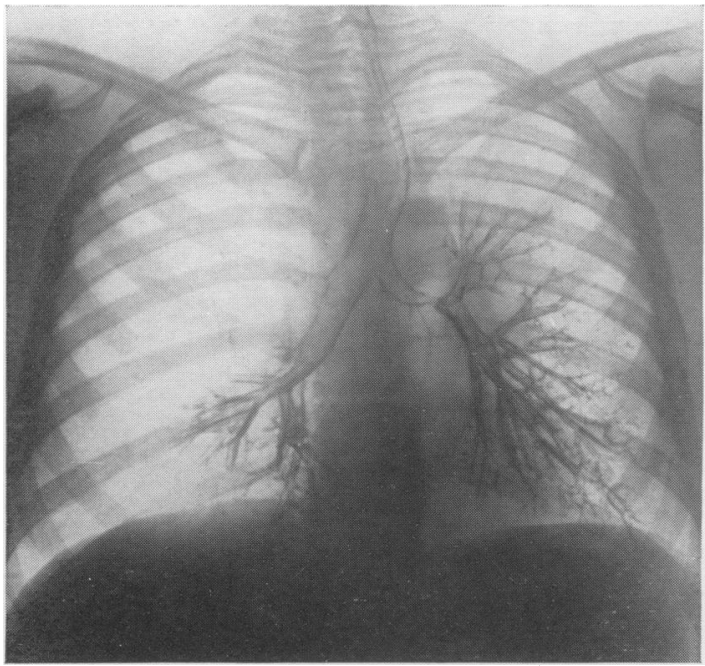

Fig. 4.-Following febrile illness and cough, patient found to have tubercle bacilli in sputum. Lung parenchyma normal radiographically and clinically. Bronchography confirmed stenosis of main bronchus seen at bronchoscopy. 


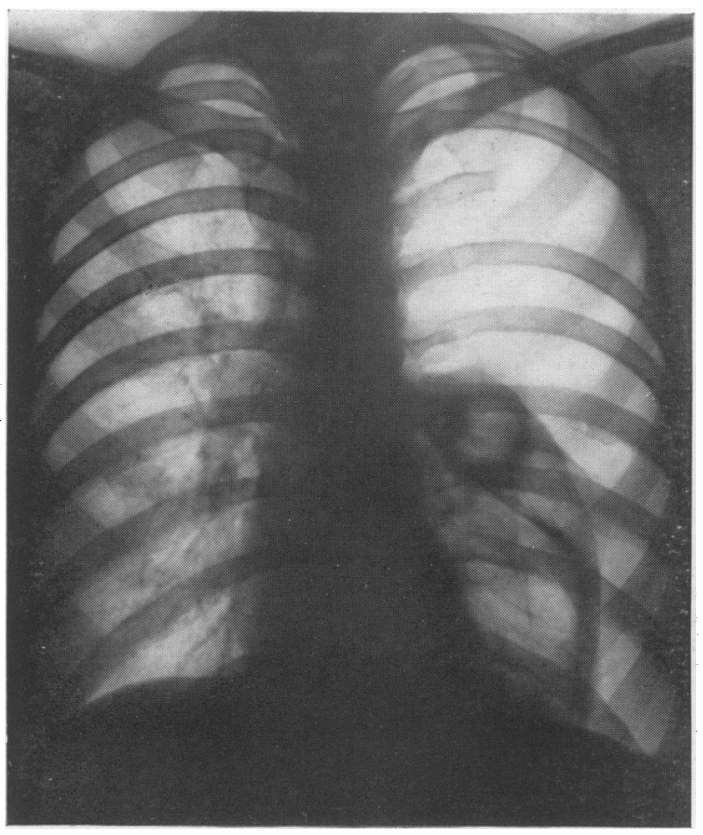

Fig. 5a.-Unilateral disease of upper lobe treated by left artificial pneumothorax. Indivisible broad apical adhesions. Pneumothorax should be abandoned or modified by additional treatment.

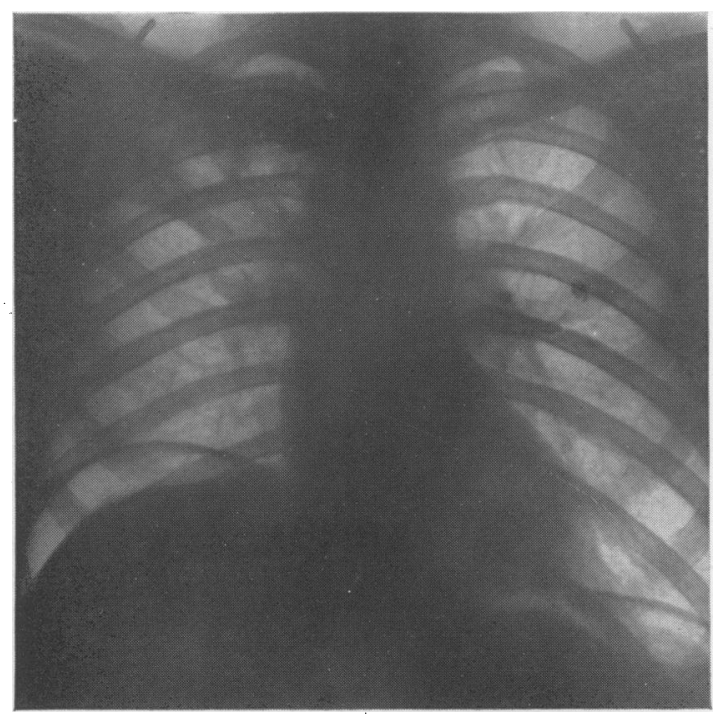

Fig. 6.-Tuberculous cavitation of right upper lobe. Pneumothorax failed and equally unsuccessful result with pneumoperitoneum and phrenic paralysis. Lobectomy produced excellent result.

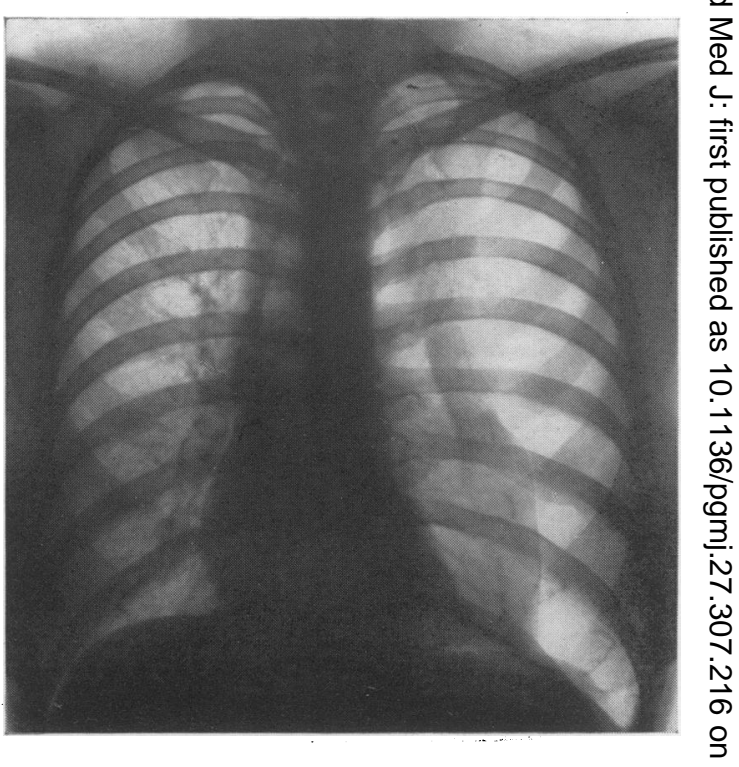

FIG. 5b.-Following extrapleural stripping and fenestra- $\vec{z}$ tion, left upper lobe retracted well but cavity remained open. Combined intra- and extra-pleural $<$ pneumothorax thus proved unsuitable and re- $\overrightarrow{0}$ section was subsequently necessary.

treatment the underlying lung may show evidence of re-expansion and, in many cases, the thick rigid layer of visceral pleura may completely incarcerate the lung. Re-expansion of the lung is $\frac{\circ}{\circ}$ important, as the pulmonary disease may be $\cong$ quiescent. If re-expansion does not occur, over- $\overrightarrow{\overrightarrow{0}}$ expansion of the opposite side with resulting 3 emphysema may follow, seriously handicapping the patient by dyspnoea and exposing him to the risk of the development of a cor pulmonale. 윽 Re-expansion of the underlying lung may follow on decortication of the pleural lining.

One must also consider the question of $a$ tuberculous empyema, which can be regarded as a $\frac{\text { 의 }}{3}$ cold abscess in the pleural cavity. These patients have usually undergone repeated chest aspirations, the pus obtained from the cavity sometimes show $-\frac{7}{0}$ ing the presence of tubercle bacilli and sometimes being sterile.

$\mathrm{X}$-ray examination of the chest should be re- $N$ peated when the pleural cavity has been completely emptied by aspiration. If any query arises as to the state of the lung parenchyma, bronchography should be performed. If all the sputum tests are negative and the lung appears unaffected radiographically the entire pleural lining can be removed. $\cdot$ The haemorrhage from the chest wall after the stripping is usually controllable. After this de- $\overrightarrow{\mathbb{D}}$ cortication the underlying lung tissue, if healthy, $\frac{?}{\mathbb{Q}}$ will re-expand and obliterate the pleural space. 


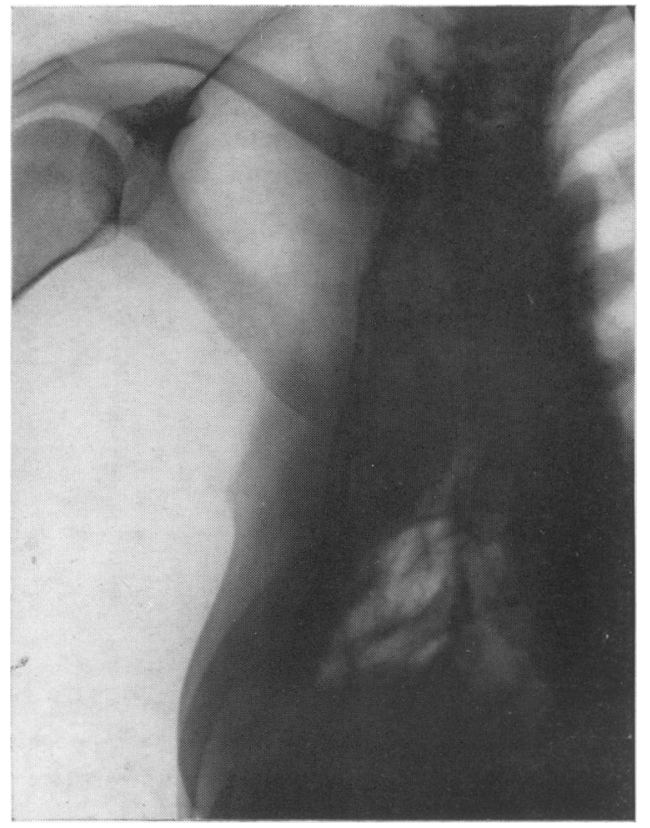

FIG. 7a.-Thoracoplasty and apicolysis for infiltration and cavitation of right upper lobe. 'Two years later sputum became positive and triangular-shaped cavity became visible under medial end of clavicle.

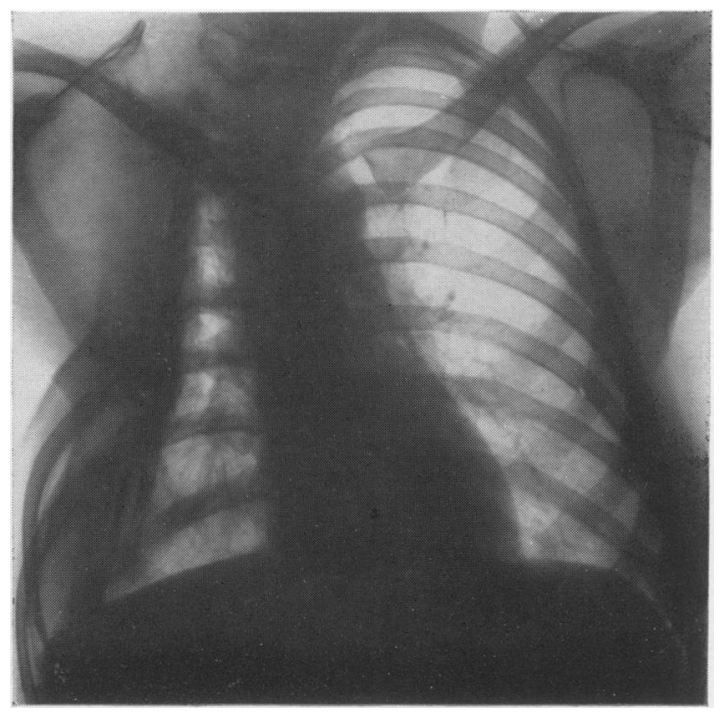

FIG. 7b.-Following right upper and middle lobectomy under previous thoracoplasty all the symptoms disappeared. Compressed upper lobe contained large chronic tuberculous cavity. The lower lobe expanded well after resection.

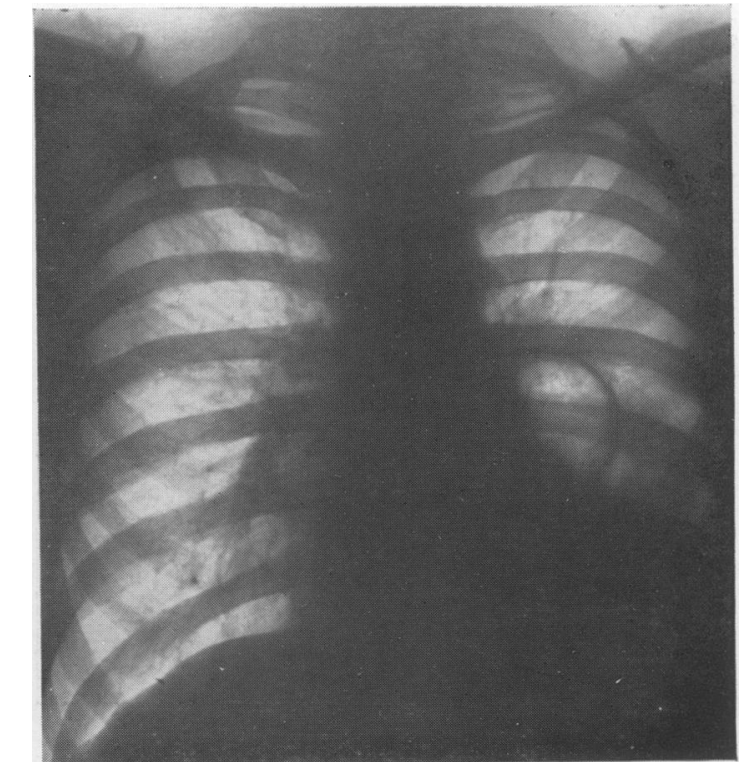

FIG. 9.-Large cavity stated to be situated in hilar region of left lung. In fact cavity is in apex of lower lobe. Pneumothorax, phrenic paralysis and pneumoperitoneum unsuccessful. Resection indicated. 


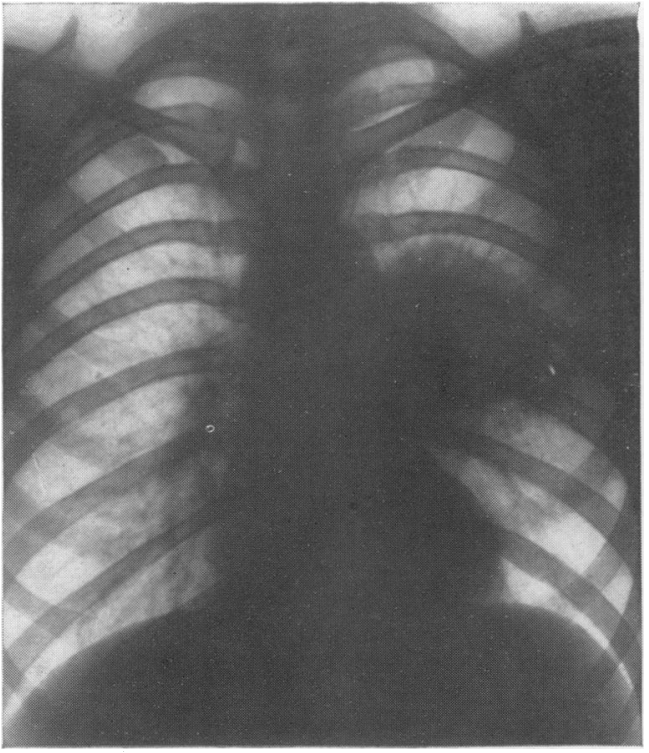

Fig. roa.-Large rounded shadow in mid-zone of left lung, believed to be a carcinoma. Resection showed this to be a tuberculoma.

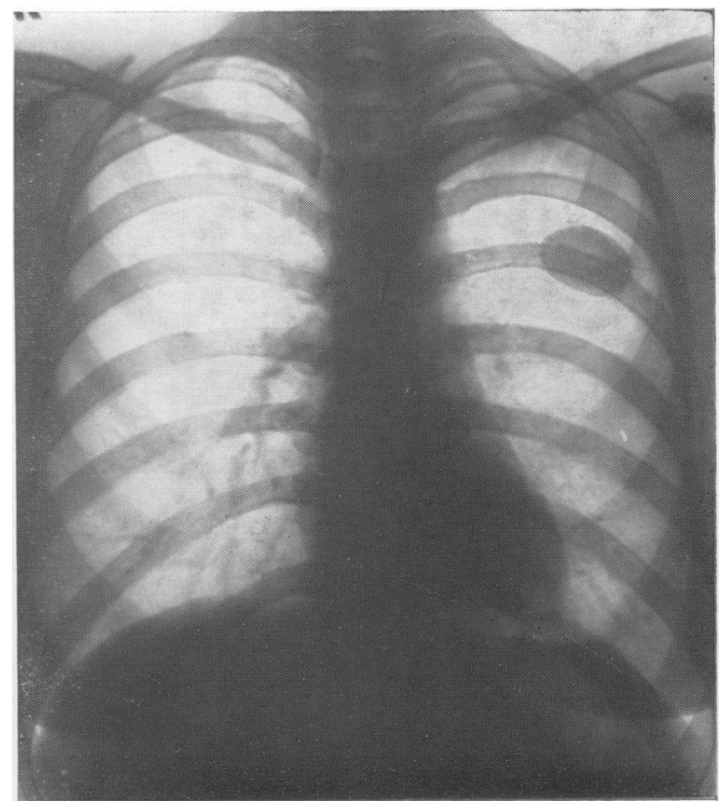

Fig. Iob.-Left artificial pneumothorax treatment for tuberculous cavity. Pneumothorax maintained for three years. Cavity visible in second interspace reappeared when pneumothorax abandoned. Resection necessary. Specimen showed encapsulated fibro-caseous focus suggestive of breaking down tuberculoma.

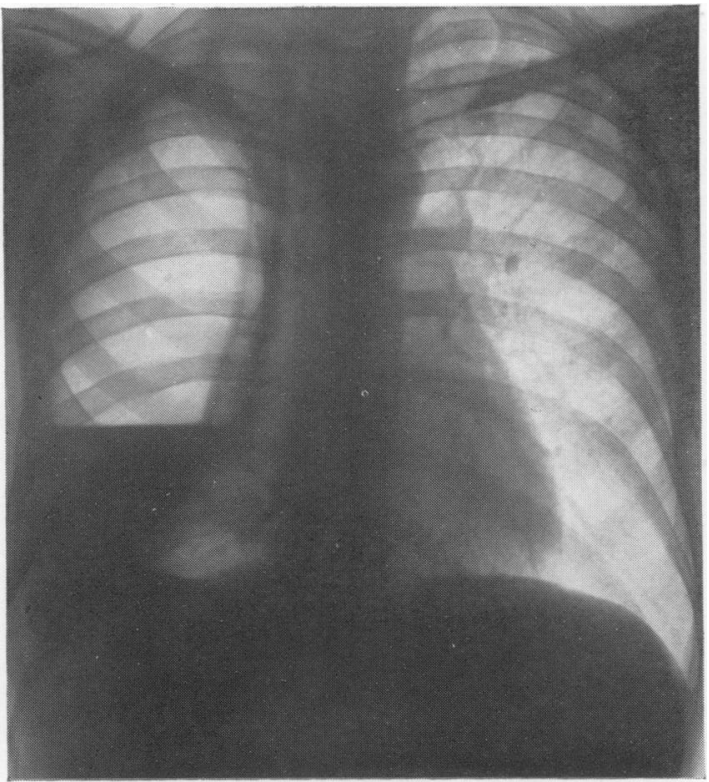

Fig. Ir.-Tuberculous empyema complicating artificial pneumothorax. Pulmonary disease quiescent and patient required pleurectomy. Lung re-expanded.
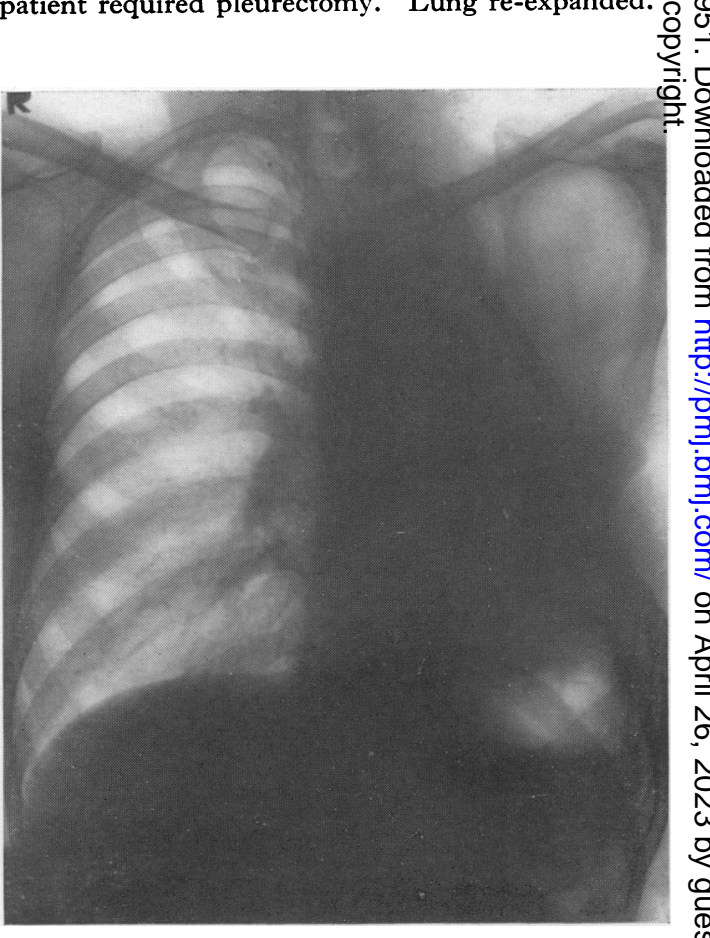

Fig. 12.-Thoracoplasty performed for left upper lobe tuberculous cavitation. Two years later repeated $\overline{0}$ severe haemoptyses occurred. Bronchography $\overrightarrow{\mathbb{D}}$ showed extensive bronchiectasis of both lobes. No $ᄋ$ evidence of active tuberculosis in upper lobe. Film $\stackrel{\mathbb{Q}}{\Omega}$ shows appearances after pneumonectomy. 
If the empyema is associated with lung disease it may be necessary to resect the affected lobe or even the entire lung in conjunction with the pleural resection.

\section{Tuberculosis and Carcinoma}

The incidence of carcinoma is not higher in tuberculous patients than in the non-tuberculous population.

Resection of the lung is a matter of urgency in cases which present the two conditions simultaneously. The general condition of the patient should be carefully assessed as a lobectomy may be a less hazardous procedure than a pneumonectomy.

\section{Bronchiectasis Complicating Tuberculosis}

Bronchiectasis may give rise to symptoms which necessitate surgical treatment even though the tuberculous disease has become quiescent spontaneously or as a result of treatment. Thus resection of the upper lobe may be indicated in cases of arrested chronic fibroid tuberculosis of the upper zone if the residual bronchiectasis is giving rise to adverse symptomatology.

Stricture of the bronchus may result from ulceration of tuberculous lymph nodes. Secretions are retained in the lobe beyond the stricture and the resulting bronchiectasis is best treated by pulmonary resection.

Atelectatic pulmonary tissue is often a precursor of bronchiectasis. The so-called 'ideal pneumothorax cases' included those cases in which the diseased area was completely atelectatic, while the normal portion of the lung remained aerated but partly compressed. Patients in this category remained well with a negative sputum during the time that the pneumothorax was maintained.

Various authorities have stated that tuberculosis heals with atelectasis. This is often true, the proviso being that the atelectasis often results in bronchiectasis. When this 'ideal pneumothorax' is abandoned new symptoms may develop as the lung re-expands. The cough, sputum production and haemoptyses of bronchiectasis soon provide a picture similar to that caused by the tuberculosis. The patient is therefore in the unhappy position of requiring a resection of the lobe of the lung at the end of a three- to five-year course of treatment. Much time and invalidity would have been spared him had the lobectomy been performed initially.

\section{Uncontrollable Haemorrhage}

Resection of lung tissue may be urgently called for if an uncontrollable haemorrhage occurs. If the usual measures of controlling haemorrhage have failed the patient's general condition may be very poor. The decision to perform a resection thus becomes a difficult one and many transfusions may be necessary. The extent of the tuberculous involvement must be carefully assessed as the inhaled blood clot may radiographically resemble 'tuberculous infiltration,' and this may suggest that more extensive disease exists than is actually the case. Fortunately, although the occurrence of haemorrhage is frequent, the problem of urgent resection does not often arise.

\section{Scarcity of Hospital Accommodation}

The scarcity of hospital accommodation provides a very real and important indication for lobectomy in a social context. The world-wide shortage of hospital beds inclines one to favour methods which set patients on the road of recovery shortly after the institution of the treatment. Pulmonary resection is a clear example of this and not only does it allow of a speedier recovery for the individual patient, but it ensures adequate treatment for a very much larger number of cases as more hospital beds become available.

\section{Pre-Operative Assessment of the Patient}

Pulmonary resection is a major operative procedure and therefore the general condition of the patient and the indications for surgery must be 8 carefully assessed before this line of treatment is adopted. 'The patient should be under observationo by the surgeon for two months prior to operation, during which time a careful record should be kept of the temperature, the body weight and the blood sedimentation rates. A record should be obtained of the reaction to exercise. The patient should be up for at least six hours each day, allowing definite time periods for slowly walking about. At the commencement of the observation period he should be walking about for five minutes a day, increasing daily to two hours walking at the end of two months.

This is contrary to the accepted views which stress pre-operative rest. During any type of pulmonary resection an enormous physical strain if placed on the circulatory and respiratory systems so the patient, and the operation may last several hours. One would not expect anyone to complete a few hours of strenous exercise after a long period of bed rest, and similarly no patient, in my opinion, is in a fit state to undergo this operation after having been confined to bed for a few months.

The operation should be postponed if there is evidence clinically or radiographically that the disease is in an active phase. Radiographic investigations at four-weekly intervals should be carried out during the observation period, and the films should be compared with those taken previously. Any sign of extending infiltration is a contraindication to operation. One must re- 
member that the disease is usually more extensive than is demonstrated by the X-ray films.

Three special investigations should always be performed prior to resection.

\section{Vital Capacity as Measured by Means of a Spiro- meter}

A vital capacity adequate for a small person may be completely inadequate for a large individual. A resection should not be performed if the vital capacity is under 1,500 cc. in a patient who weighs $125 \mathrm{lb}$., or under $1,800 \mathrm{cc}$. in a patient weighing from 125 to $175 \mathrm{lb}$., or under $2,000 \mathrm{cc}$. in those patients weighing more than $175 \mathrm{lb}$. It is not enough to estimate the vital capacity in the usual manner, which measures the amount of air exhaled after a maximum inspiration.

Many individuals have a vital capacity of almost $2,000 \mathrm{cc}$. when emphysema or bronchospasm is present. They may take 15 to 20 seconds to blow all this air into a spirometer. After about five seconds they may strain and exhale a portion of their residual air, and it is this extra amount which leads to the false reading of about $2,000 \mathrm{cc}$.

A reasonably accurate reading of vital capacity will be obtained if the patient is requested to exhale as rapidly as possible and if the examiner disregards any air exhaled after the initial period of five seconds.

The discovery of a low vital capacity is a definite contraindication to resection as the surgical procedure will diminish it still further even up to the point of respiratory insufficiency.

\section{Bronchography}

Bronchography should be performed about a month before the operation. We know that the tuberculous disease is usually more extensive than is shown by ordinary $\mathrm{X}$-ray examination, but the instillation of iodized oil may do more than merely confirm this, as it will show coexistent areas of cavitation and bronchiectasis.

As a total filling of the affected lung is necessary the investigator must exercise both skill and patience. Bronchograms will indicate the extent of the tuberculosis and thus the magnitude of the resection, and will also demonstrate the exact localization of the disease process. Ordinary iodized oil eventually percolates into the alveoli. If the oil is thickened with sulphathiazole or sulphanilamide powder in the proportion of $5 \mathrm{gm}$. to $20 \mathrm{cc}$. of oil, the patient is usually able to expectorate all the mixture within a short space of time.

Some authorities believe that the iodized oil leads to a spread or flare-up of the tuberculous disease, but this has not been my experience.

The technique used in bronchography is not important as long as all the affected lung is filled and good X-ray films are secured. The exact limits of the lung can be outlined by bronchography. Lesions which may appear intrapulmonary on ordinary films may be shown to be extrapulmonary, i.e. in the pleural space or membranes, on bronchography.

\section{Bronchoscopy}

Inspection of the bronchial tree should always be carried out to exclude the presence of ulceration. Division of a bronchus at the site of an ulcer will lead to the breakdown of the line of repair and subsequent contamination of the pleural cavity.

Radiographically the disease may appear to be localized to one particular lobe, the remaining lobes appearing unaffected. Findings at bronchoscopy may complicate the issue by revealing tuberculous ulceration of the bronchi supplying the other lobes. Consequently the necessary resection may be wider than was originally contemplated.

If ulceration in the region of the carina is demonstrated all ideas of pulmonary resection must be temporarily abandoned.

\section{Surgical Technique}

The programme for the treatment of tubercu- $\varnothing$ losis by pulmonary resection may conveniently be divided into four stages.

I. The period of preparation.

2. The operation of resection.

3. The post-operative period.

4. The period of rehabilitation.

The final stage is beyond the scope of this article as it deals with the patient who is sufficiently restored physically to be sent to a sanatorium. After observed and adequate conditions of rest and diet and the performance of graduated exercises in the sanatorium he may be returned to his normal place in society.

\section{Pre-Operative Preparation}

The patient should receive $\frac{1}{2} \mathrm{gm}$. injections of streptomycin intramuscularly morning and evening for 14 days prior to operation. This is designed to prevent any spread of the disease during the pre-operative and operative periods of the treatment. Since the introduction of streptomycin surgical procedures are rarely followed by disease extension.

P.A.S. usage must be controlled as it has been shown that it leads to a bleeding tendency. If the patient has been receiving P.A.S. therapy it should be discontinued for the 14 days before operation. If necessary this treatment can be resumed after the recovery of the patient.

Twenty-four hours before the operation penicillin in doses of 500,000 units should be combined 


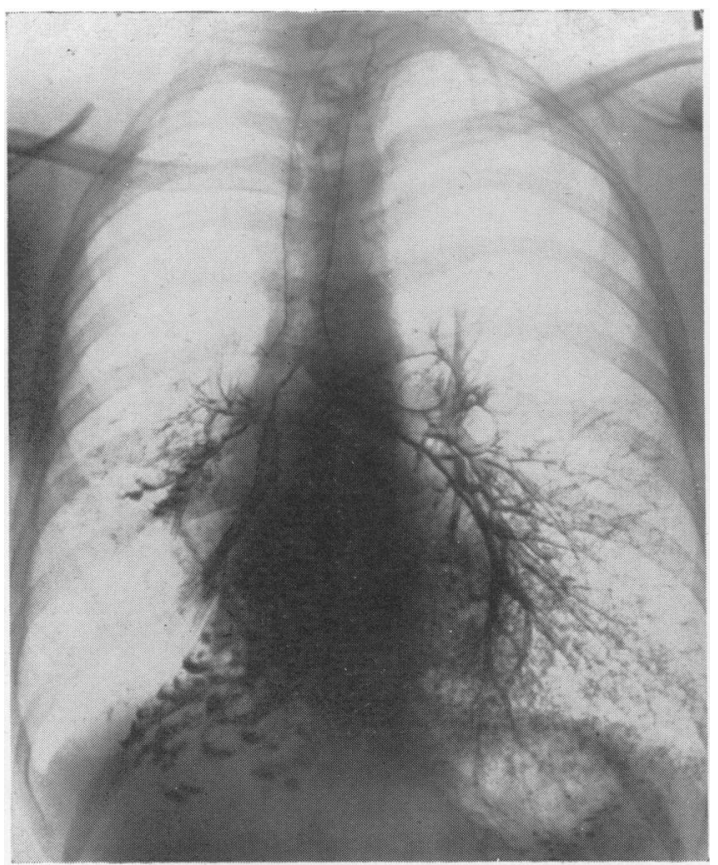

FIG. 13.-Stricture of stem bronchus immediately below upper lobe bronchus on right side. Bronchiectasis of lower lobe. Examination following lobectomy showed cause of stricture to be due to tuberculous gland.

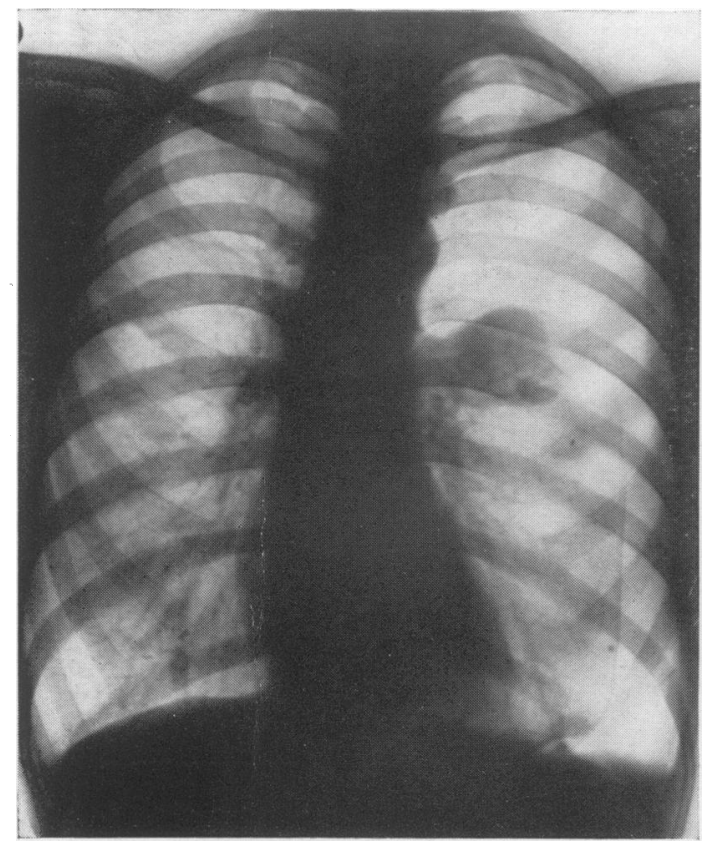

FIG. I4b.- Similar case except that in spite of atelectasis of upper lobe of left lung this also showed presence of cavities in this collapsed lobe. Following lobectomy the specimen showed numerous caseating areas.

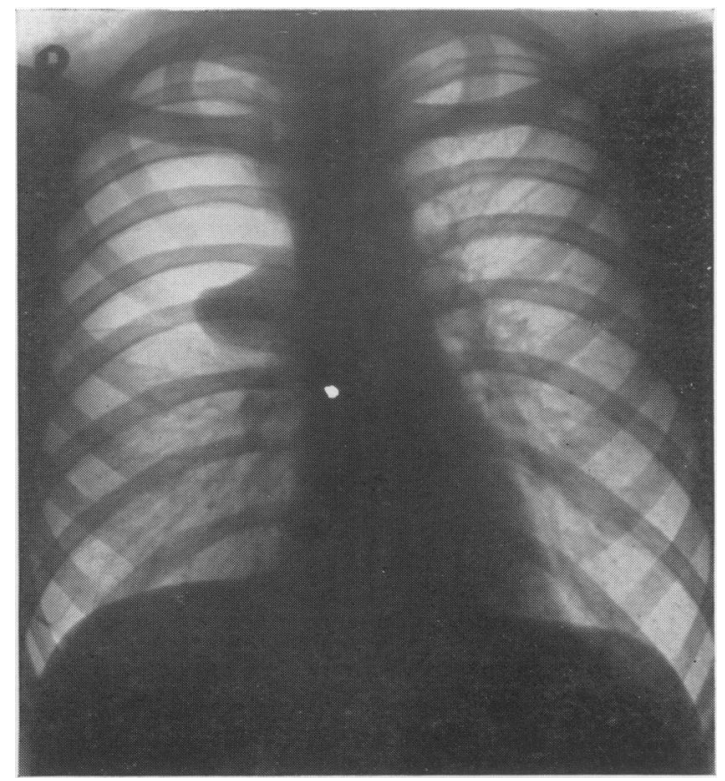

FIG. I 4a.-Following right artificial pneumothorax, atelectasis of upper lobe occurred. In spite of absence of symptoms lobectomy was indicated as this lobe failed to aerate when the pneumothorax was being abandoned.

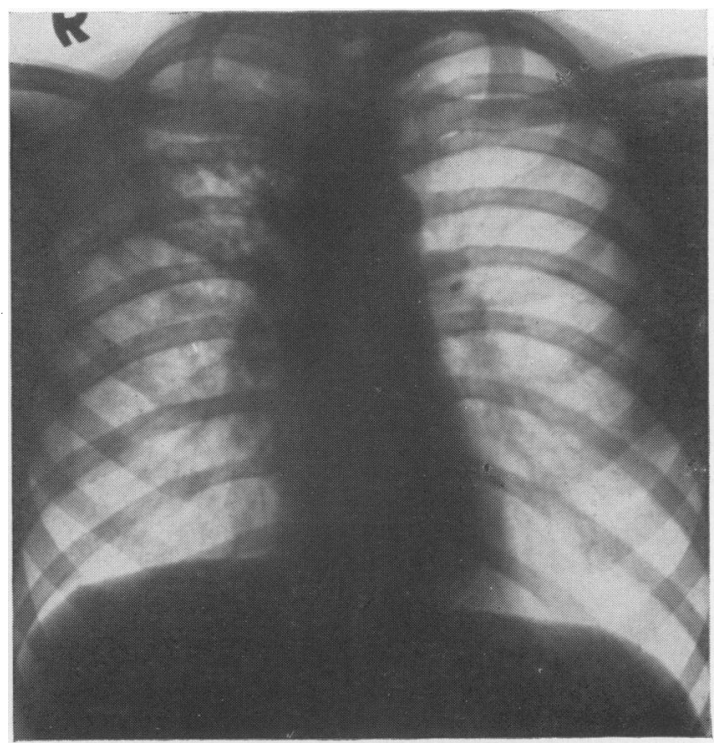

FIG. 15.- Infiltration and cavitation at first confined to upper lobe of right lung. Disease extended into lower lobe during period of observation. Pneumothorax failed. Resection is absolutely contraindicated at this stage. Pneumoperitoneum and right phrenic paralysis is indicated as well as use of streptomycin and P.A.S. 


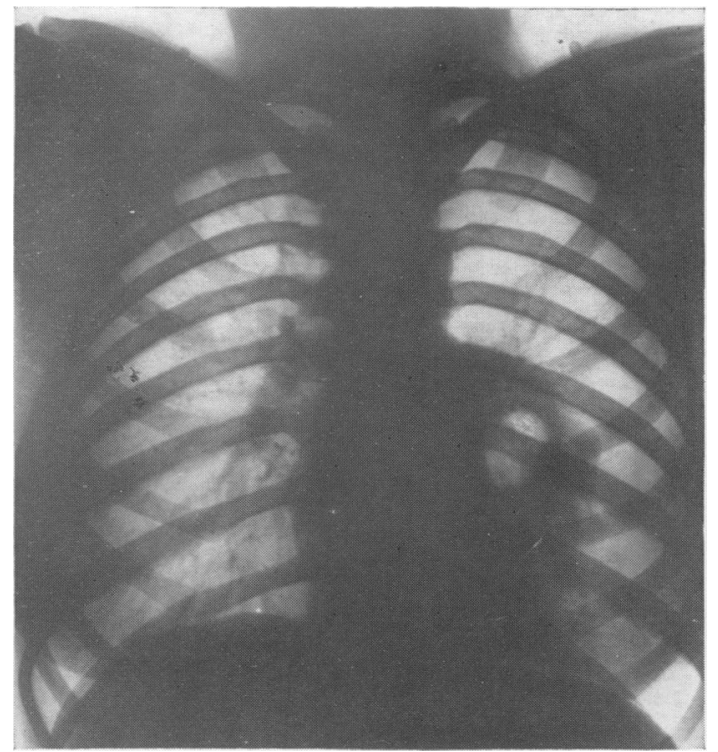

FIc. I 6a.-Infiltration and cavitation of the lower lobe of left lung. After pneumothorax the cavities in the apex of the lobe remained open.

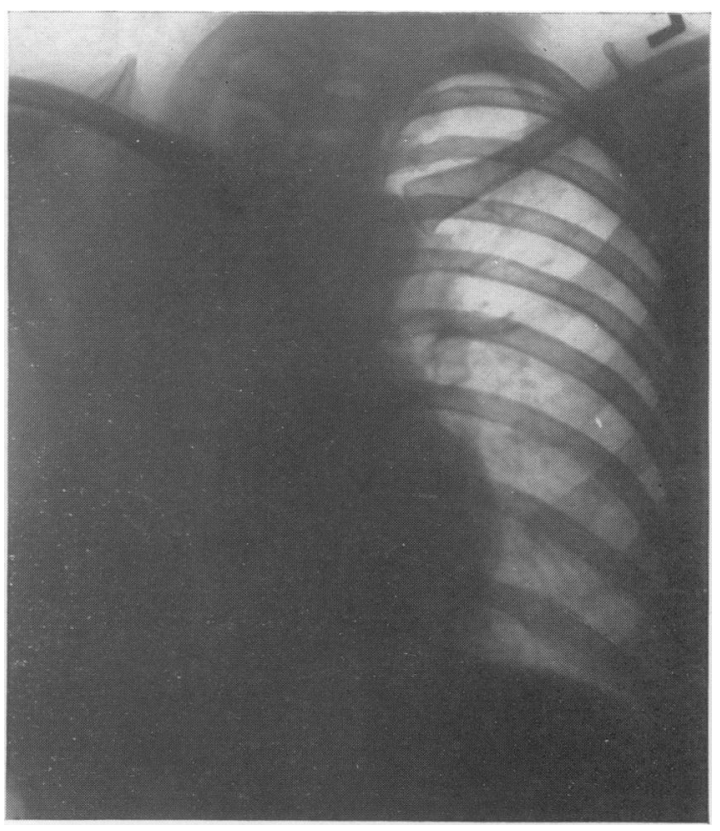

FIG. I 7.- Post-pneumonectomy thoracoplasty because of rapid absorption of pneumothorax and movement of mediastinum towards operation side. Ribs No. 2 to No. 7 removed.

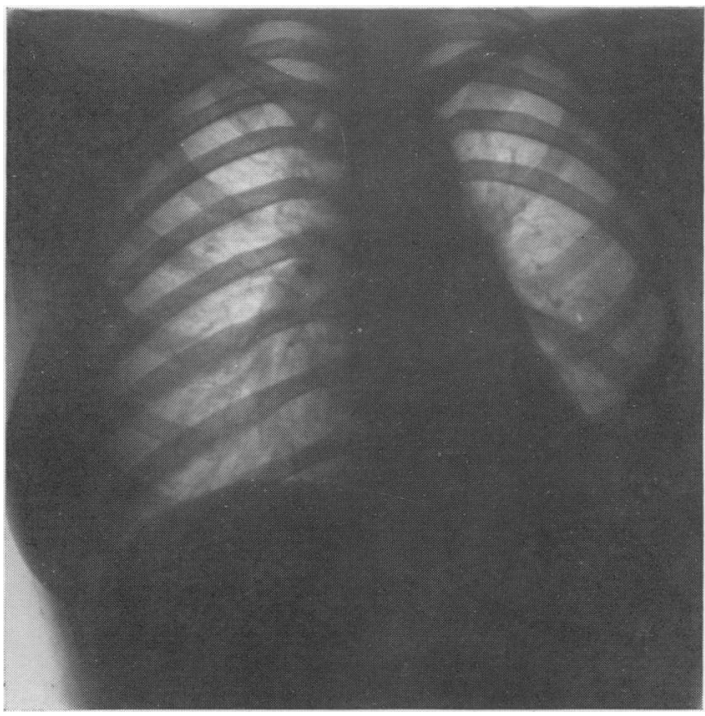

Fig. I6b.-Following left lower lobectomy the upper lobe expanded, almost filling pleural space. Healing rapid and uncomplicated.

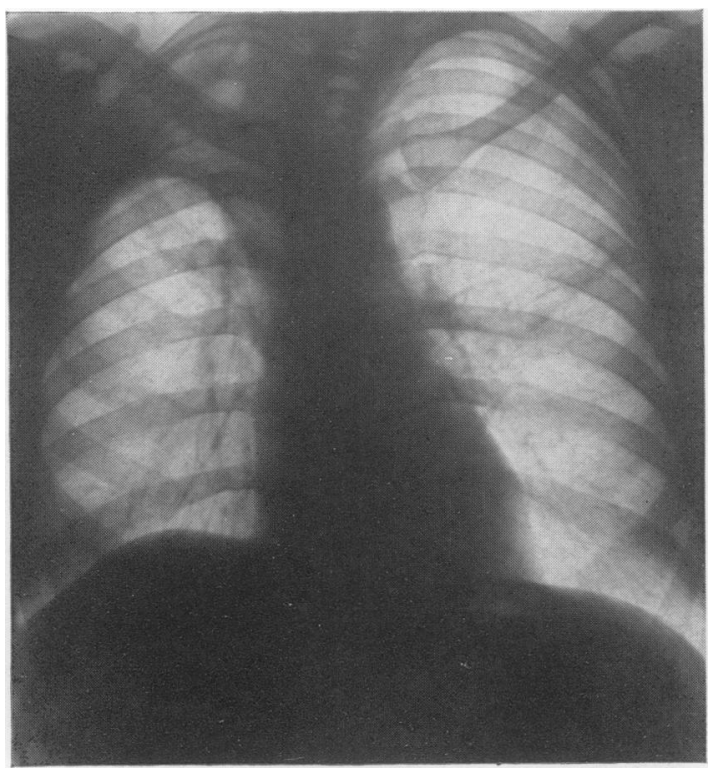

FIG. 18.-Bilateral disease. Right upper lobe removed and disease in left mid-zone unchanged. Film shows residual pneumothorax and effusion following upper lobectomy, no attempt being made to empty the pleural space completely. 
with the streptomycin administration. These two antibiotics are of vast importance in the prevention of pyogenic infection and in the retardation of the activity of the tuberculous process.

\section{The Operation of Pulmonary Resection}

Premedication of morphine sulphate, $\frac{1}{4}$ gr., or omnopon, $\frac{1}{3} \mathrm{gr}$., is administered half an hour before the operation. The nasopharynx and throat are sprayed with a solution of 10 per cent. cocaine hydrochloride, the patient having been given a cocaine sensitivity test at some previous time. A small quantity of pentothal sodium is injected into the intravenous drip saline, which should be set up before the operation.

The anaesthetist then passes an intratracheal tube, packs off the pharynx, and pentothal and curare anaesthesia is commenced, the lungs being ventilated by oxygen.

The patient is placed in the face-downwards position as soon as anaesthesia is satisfactorily established. The side of the chest which is to be operated upon should be tilted slightly upwards. A long postero-lateral incision is made round the vertebral border of the scapula extending from the spine to a position situated well anteriorly. The surgeon, who has full cognisance of the site of the disease, should then remove the rib appropriate to the lobe or segment to be resected. Resection of the fifth rib ensures accessibility to the entire pleural space. It is difficult, however, to remove an upper lobe after a seventh rib resection.

The pleural cavity should then be entered and any adhesions attaching the lung to the chest wall should be divided. Adhesions, which are usually present, are in many cases dense and vascular, and it is advisable to clamp them, divide them and then seal them with an electro-cautery.

\section{Dense Pleuro-Pulmonary Adhesions}

The lung may be firmly and inseparably attached to the parietal pleura. The attachment generally occurs over old cavity areas and there is always the risk of tearing into the lung during any attempt at separation from the pleural lining. If this accident of opening into such cavitated areas with its attendant contamination of the pleural space occurs, it is advisable to carefully wash out the pleural cavity with saline when the resection is completed, in an attempt to minimize the danger of empyema formation.

An extrapleural separation of the lung from the chest wall over these firmly-adherent sites will avoid this hazard, leaving the residual portion of the lung with an attached cap of parietal pleura. The bare area left by the removal of the parietal pleura may bleed a little, but this bleeding is easily controlled by the application of hot swabs maintained for some time in the requisite position. If, however, there is a slow oozing of blood from the raw surface, the blood loss should be replaced via the intravenous drip apparatus.

The operation of lobectomy involves the exposure of the pulmonary hilum and the opening of the interlobar fissures. This procedure may become very difficult if the entire lung is covered by a dense membrane, which makes it appear to be a single sheet of tissue undifferentiated into lobes. In such a case the mediastinal surface of the lung should be exposed. This exposure will normally allow of a demonstration of the interlobar fissures and lobar differentiation.

\section{Types of Resection}

The operations of pneumonectomy, lobectomy and segmental resection of a lobe are the methods employed. As the operations are identical to those performed for bronchiectasis, a general description will not be given in this paper and only special features of the operative technique will be discussed.

\section{Determination of the Extent of the Disease}

As soon as the lung has been freed from all attachments to the thoracic wall, the surgeon should determine the extent of the disease. Radiography may have indicated the presence of the disease in a particular lobe, but actual palpation and inspection may reveal a far greater degree of pulmonary involvement.

When the extent of the disease has been determined the resection of the affected segment, lobe or lung can be performed according to the methods of resection employed in the operative therapy of bronchiectasis.

\section{Repair of the Bronchus}

Many different means of bronchial repair have been described, but the method of choice is that in which the minimum number of sutures is employed. Care should be taken to avoid devitalizing the stump of the bronchus by the use of clamps, as this predisposes to fistula formation. The divided bronchus should be treated with carbolic acid and alcohol in the manner used for the treatment of the appendix stump. This aids in the sterilization of the end of the stump, and destroys the mucous membrane at that site, leading to rapid healing with the formation of a satisfactory cul-de-sac.

The first step in the repair of the bronchus is to fold the bronchus on itself and to insert two sets of mattress sutures. These sutures should be of a non-absorbable material, such as nylon thread, and they should interlock and be firmly tied. When the first suture has been inserted the anaesthetist should be instructed to test the air tightness by 
increasing the pressure of the anaesthetic gases. If an air-tight closure has been obtained the second suture should be inserted and equally firmly tied.

After pneumonectomy the bronchial stump should be short enough to allow of its retraction into the mediastinum, where it can be adequately protected by the aorta or the azygos vein.

A small amount of a powder mixture of penicillin, streptomycin and sulphanilamide should be sprinkled on the stump prior to the repair of the mediastinal pleura. After lobectomy the bronchial stump should first be dusted with antibiotic powder and then covered either by means of a pleural flap or by the overstitching of the adjacent tissues.

\section{Phrenic Paralysis}

Phrenic paralysis has been undertaken by many surgeons in order to reduce the size of the residual space after resections and to delay the full expansion of the remaining lung tissue. In my opinion it is a thoroughly unsound procedure, for two reasons. First, the paralysis of the diaphragm on one side will interfere with the cough mechanism: A patient who has had a resection must be encouraged to cough up the secretions which have collected, especially from the side which has been operated upon. This becomes extremely difficult when the phrenic nerve has been crushed.

Even more important, however, is the fact that phrenic paralysis causes paradoxical movement of the halves of the diaphragm. There is a rise in intrapleural pressure on the unaffected side during coughing and a corresponding fall in pressure on the side which has been surgically treated. The force of the pressure will thus be borne by the repaired bronchial stump with the grave risk of the formation of a bronchial fistula. The force of a cough is directed against the glottis when both sides of the diaphragm are active.

The complication of bronchial fistula has not been encountered in this series of cases.

\section{Drainage of the Pleural Cavity}

The pleural cavity should be carefully washed out several times with a saline mixture before the operation incision is repaired. During this procedure the lungs should be distended to prevent any aspiration or leak into the bronchial system. This will demonstrate the air tightness of the repaired bronchus.

An intercostal drain leading into an underwater drainage bottle should be inserted just above the diaphragm in a postero-lateral position. The introduction of a Foley catheter, which prevents infection gaining entry via the tube, avoids the necessity of transfixing the tube by means of a stitch or safety pin. The balloon-like portion of the catheter is filled with a penicillin solution which is allowed to run out before the catheter is removed.

The catheter is generally withdrawn 48 hours after operation. If, however, a portable X-ray examination of the chest reveals a large residual effusion which is still draining, the catheter must be retained in position for a longer period.

\section{Post-Operative Bronchoscopy}

The manipulation during resection often forces secretions into the bronchi from the lungs. These secretions may lead to considerable respiratory embarrassment, as the patient cannot expel the mucus beyond the glottis. This is particularly apparent after phrenic paralysis, which depresses the cough reflex.

A post-operative bronchoscopy, which will establish the condition existing in the bronchi, should be regarded as a routine measure after resection. There is no greater risk attached to a post-operative bronchoscopy than to a bronchoscopy performed on a properly anaesthetized patient during the process of investigation. The warning should be given, however, that the passage of a bronchoscope in an almost conscious un anaesthetized patient may well lead to severe shoc and death.

A bronchoscopy may become necessary a day oD so after operation as the presence of unexpectorated thick, tenacious sputum may cause severe distress. The bronchoscopy, which is performed on the patient under local anaesthesia in his bed in the ward, gives instant relief.

\section{Treatment of the Residual Space}

For many years surgeons have been concerned with the fate of the residual space. This problem is of greater importance following on a pneumonectomy as, after lobectomy, the remaining lobe or lobes expand and aid in the obliteration of the space. Two important points should be considered:

I. Overexpansion emphysema. If the unaffected lung expands to such a degree that it encroaches on the operated side, overexpansion emphysema develops. The patient becomes intensely dyspnoeic ? and manifests the complete picture of chronic $N$ bronchitis and emphysema. This complication, N which may occur after pneumonectomy, carries with it a grave prognosis.

2. Exacerbation of quiescent tuberculous disease. This complication may be seen after a lobectomy $\stackrel{\mathbb{Q}}{?}$ where a reactivation of quiescent foci may result from the expansion of the remaining lung tissue. The actual effect of the emphysema which occurs in the remaining lung tissue is usually negligible. 
Many devices have been employed in attempts to prevent the onset of overexpansion emphysema. Plastic moulds, shaped like a lung, have been inserted into the pleural cavity. Leucite balls and olive oil mixtures have been used to keep the mediastinum in its central position.

We have found that the most efficacious method is to maintain the pleural space with air in exactly the way used in pneumothorax threatment. One month after the pneumonectomy operation about $25 \mathrm{cc}$. of a half-saturated solution of urea is placed in the pleural space. This causes a tissue reaction with the formation of a firm, fibrous tissue layer over the entire area. Refills to maintain the pneumothorax may be given every six or eight weeks.

By this process of maintaining a small pneumothorax the mediastinum remains in a fairly central position and shows very little tendency to be disadvantageously displaced. The residual lobe is slowly allowed to expand over a period of a year or more.

These methods of treatment have proved to be very effective. In a remarkably small number of cases after pneumonectomy the pneumothorax has absorbed very quickly and the sound lung has shown early signs of encroaching on to the operated side. An upper thoracoplasty has been performed, leaving the first rib intact. This has avoided the dangers of overdistension emphysema.

Many clinics have recommended routine postpneumonectomy thoracoplasties, and the thoracoplasty may even be commenced at the time of the pneumonectomy operation. The use of thoracoplasties as a routine procedure has not been considered necessary in the cases under review.

\section{Complications of Pulmonary Resection}

The possible complications of resection treatment may be attributable to the actual operation or to the disease process itself.

\section{Haemorrhage}

Considerable blood loss may attend the combined operation of pneumonectomy and pleurectomy. The stripping of the thickened pleural membrane from the thoracic wall exposes numerous bleeding surfaces. Adequate supplies of blood should be available during the operation. As a rough guide one may estimate the amount of blood lost as being equal to double the quantity of blood sucked up by the suction apparatus. It follows then that this amount should be replaced during the operation.

It is important to remember that the blood loss may continue after the completion of the operation, and a blood transfusion should be given to replace the quantity which has drained into the drainage bottle.
In my opinion P.A.S. treatment should be discontinued about I4 days prior to operation. During this period the patient should receive daily injections of vitamin $K$. The continued administration of P.A.S., which is supposed to delay the clotting time, may lead to serious postoperative haemorrhage.

\section{Operative Shock}

A large degree of shock is not often encountered during a straightforward lobectomy operation, but profound shock may be seen where pleural stripping has been done. If the lung is densely adherent to the chest wall and a pneumonectomy is to be performed, the stripping should be carried out while the anaesthetist gives intravenous procaine hydrochloride as a drip injection. For this purpose ro cc. of I per cent. procaine should be added to roo cc. of normal saline solution.

Shock is not likely to occur after the pleural stripping and the dissection of the hilum have been completed.

\section{Infection of the Pleural Cavity}

Infection of the pleural cavity may be due to pyogenic organisms or to tubercle bacilli. The pleural cavity may be contaminated at the time of operation by accidental entry into a tuberculous cavity. Careful and liberal washing of the pleural cavity with saline solution should effectively deal with all contamination and ensure primary healing.

Tuberculous infection of the pleural cavity may, however, only occur several days after the operation. It is probably more correct to state that the infection is recognized at that stage as the contamination probably had occurred at the time of operation.

In many cases, infection by tubercle bacilli was only seen after a difficult resection where the lung was firmly attached to the chest wall and pieces of pulmonary tissue had probably been left in their attachment to the thoracic cage.

Tuberculous empyemata were not seen after straightforward pulmonary resections and the low incidence of this complication has been attributed to the careful repair of the bronchial stump.

Analysis of the post-operative results reveals the fact that there is more likelihood of a tuberculous empyema developing after a segmental resection for infiltrated and cavitated areas. As an example of this I may cite a case in which the apex of the lower lobe was involved in a cavity of the upper lobe. A resection of the upper lobe and the apex of the lower lobe was performed and was followed in ten days by a tuberculous empyema. I consider that the operation of segmental resection should be reserved for cases of tuberculomata and very localized circumscribed cavities. It is not wise to resect 
a segment when infiltration is present, as the plane of division may penetrate through macroscopically invisible tuberculous tissue.

\section{Bronchial Fistula}

Signs of bronchopleural and bronchocutaneous fistulae may present on the seventh to the tenth day after operation. Generally the patient coughs up the contents of the pleural cavity which, initially, consist of blood-stained material.

This is a rare complication and has only once been seen in a series of over 200 lobectomies and pneumonectomies performed for diverse conditions. It may be prevented by the careful closure of the bronchus and by the application of a pleural graft over the stump.

Rapid healing has been obtained. by the closure of the bronchus by infolding and the use of a minimum number of sutures. Usually two mattress sutures with nylon thread are sufficient to give the airtight closure of the bronchus, which is reinforced by the application of a pleural flap over the repaired stump. The patient should be warned that he may cough up stitches many months after the operation, as they are nonabsorbable, and the sudden appearance of a small stitch and a trace of blood-stained sputum may be most alarming.

If a bronchial fistula develops, immediate adequate pleural drainage through the chest wall must be provided to prevent the aspiration of blood and serum into the pulmonary system. The remaining lung tissue has usually become compressed and covered with a thick pyogenic membrane. The immediate drainage of the pleural cavity encourages the expansion of the remaining lobes, which obliterate the pleural space. In the past the use of the lung tourniquet was invariably followed by fistula formation on about the tenth day after operation.

\section{Spread of the Tuberculous Disease}

Spread of the disease is naturally the most serious complication of the surgical treatment of tuberculosis. I have not been able to accept the theory of the contralateral spread of tuberculosis via the bronchi. This view states that the manipulation of the lung during operation favours the inhalation of infected material into the unaffected side. This cause of contralateral spread should operate similarly while the patient is in deep sleep.

Contralateral spread is not frequently seen. Even when copious quantities of infected sputum issue from the upper lobe the lower lobe of the same side is seldom affected. In my opinion the contralateral spread is probably a result of infected emboli containing tubercle bacilli being ex- pelled into the blood stream and reaching the opposite lung via the pulmonary artery. The lung parenchyma acts as a filter to the bacilli and this prevents the onset of generalized infection or miliary tuberculosis.

There is also the question of exacerbation of quiescent or arrested disease. Many cases may show radiographic evidence of disease in the socalled ' sound side' of the chest. Unless pathology remains unchanged for three months there is relatively little use in performing a resection on the more seriously affected side. There is always the possibility of an exacerbation of this quiescent disease, but it has been.my experience that an adequate course of streptomycin and P.A.S. before and after operation considerably reduces the danger of this unfortunate result.

It is important to remember that no signs of tuberculosis may be seen on the sound side by ordinary radiological methods. Suspicious nodules may become apparent when softer radiographs are taken and it can be assumed in some cases, where post-operative spread is suspected, that the pathology is really due to a reactivation of these nodules. It is always a good plan, therefore, to supplement ordinary radiography of the chest with a set of 'softer' films before a resection is pero formed.

Atelectasis of the Remaining Lobes after Lobectom

Many surgeons in the past have deliberately caused adhesions before resecting a lobe for bronchiectasis, as they believed that these adhesions would prevent collapse or atelectasis of the remaining lobes of the lung. Similarly, many surgeons today, in treating tuberculosis by lobectomy, feel more satisfied if the remaining lobes are attached to the chest wall by the type of dense adhesion that one sees after prolonged pneumothorax therapy. Actually these residual adhesions do not prevent collapse, but prevent re-expansion of the remaining lobes. It is my practice to separate the remaining normal lobes from the parietes and to divide all the restraining adhesions. The anaesthestist is then requested to distend these lobes in order to be assured of their full expansion. The rest provided to the diseased lung by the pneumothorax treatment may thus become a source of embarrassment to the lung by virtue of the subsequent fixation and immobilization.

\section{Residual Effusion in the Pleural Space}

There is a grave danger of a residual effusion remaining after the operations of lobectomy and pneumonectomy becoming infected. It has been stated that if the bronchial stump is adequately repaired fistula formation is not likely, and consequently infection does not supervene. Large

\section{帝}


effusions, however, may rupture into the bronchial system if severe strain results from a bout of coughing. It is therefore important to ascertain that a minimal residual effusion exists. The drainage tube should allow all effusions to escape during the first 48 hours after operation. Subsequent to this regular 48 hourly radiographic examinations will manifest the presence of accumulating secretions. If the effusion is large enough to compress the remaining lobe or lobes, or to displace the mediastinum, the chest should be aspirated. This aspiration may involve the removal of air as well as effusion. It is not necessary to try and empty the pleural cavity as attempts to drain it completely may result in damage to the remaining pulmonary lobes from the point of the aspirating needle.

The incidence of these large troublesome effusions has not been high and aspirations have only been necessary in less than ro per cent. of cases. A mixture of one million units of penicillin and I gm. of streptomycin should be injected into the pleural space after aspiration. I, personally, believe that a small effusion serves a useful purpose, as it controls rapid overexpansion of the remaining lobes after a lobectomy. The presence of the effusion and air also helps to maintain the mediastinum in a central position after a pneumonectomy has been performed.

\section{Post-Operative Emphysema}

The problem of post-operative emphysema, which has been previously discussed, is closely bound up with the problem of the residual pleural effusion. While overdistension emphysema is not of significance after a lobectomy it is extremely important after a pneumonectomy, particularly after a pneumonectomy on the right side in which the pleural space is much larger than on the left. The prevention of overdistension emphysema must be stressed, as it may convert the patient into a complete respiratory cripple.

The mediastinum is movable for the first few weeks after pneumonectomy, and if a high negative pleural pressure exists on the side of the resection the remaining lung will over expand until it eventually displaces the mediastinum into the pleural space. Many surgeons prevent this occurrence by proceeding with an early postpneumonectomy thoracoplasty, but this is not necessary in all cases.

The maintenance of a pneumothorax on the resected side until the pleural reaction is sufficient to cause mediastinal fixation has been described. The presence of a small effusion in the pleural space aids in this process, as it leads to more rapid thickening of the pleural lining.

When the mediastinal pleura is very thick and rigid gross displacement of the mediastinum cannot occur. The small amount of displacement which results is no greater than that seen following thoracoplasty. The indications for a thoracoplasty to prevent post-pneumonectomy emphysema are quite straightforward. If rapid displacement of the mediastinum takes place, in spite of the pneumothorax refills, a thoracoplasty should be performed about six to eight weeks after the pneumonectnmy.

\section{Post-Operative Physiotherapy}

Post-operative physiotherapy should be commenced 24 hours after the operation and if the patient's condition permits he should discard the supine position as soon as possible. On the second post-operative day he should sit up with his feet dangling over the side of the bed, and on the third day he should be helped out of bed.

The remedial exercises aided by an expert physiotherapist are of paramount importance in ensuring good posture and the maximum use of the remaining pulmonary tissue.

\section{Convalescence}

It is my custom to recommend a period of six months controlled convalescence in a sanatorium. It is not always possible for the patient to arrange for this extended period of rehabilitation, even though it is offset by the fact that the actual duration of hospitalization is comparatively short. During this time careful re-education by means of graduated exercises must be carried out and frequent radiographic and sputum examinations should be performed.

We are often forced to face the problem that patients treated by resection methods are not willing to go to sanatoria for fear of ' contracting tuberculosis again.' This attitude has been seen in practically every case discharged from the thoracic surgical unit to a sanatorium. In an attempt to ease this unfortunate quandary we have tried to separate all the cases who regard themselves as free of infection, though this procedure is naturally not always possible.

\section{The Follow-up}

A routine monthly general examination and sputum test should be undertaken after the patient has left the sanatorium. A new full-sized X-ray film of the chest should be reviewed every two months. We make a point of stressing to the patients the fact that they may still have latent pulmonary tuberculosis, as this seems to encourage them to attend for regular examinations.

\section{Results}

A series of $5^{\circ}$ cases of pulmonary tuberculosis treated by resection is reviewed (Table $\mathrm{I}$ ). There 
TABLE I

\begin{tabular}{|c|c|c|c|c|c|c|c|}
\hline \multirow{2}{*}{ Operation } & \multirow{2}{*}{ Cases } & \multirow{2}{*}{ Deaths } & \multicolumn{3}{|c|}{ Contra-Lateral Disease } & \multirow{2}{*}{$\begin{array}{c}\text { Broncho- } \\
\text { Pleural } \\
\text { Fistula }\end{array}$} & \multirow{2}{*}{ Empyemac } \\
\hline & & & Present & Spread & Reactivity & & \\
\hline Lobectomy & 38 & $\circ$ & 8 & $\circ$ & $\circ$ & 0 & $0 \quad \frac{7}{6}$ \\
\hline Lobe + Segment Resection & 4 & $\mathbf{I}$ & 2 & 0 & $\mathbf{I}$ & 0 & $\frac{\bar{\sigma}}{\bar{\rho}}$ \\
\hline Pneumonectomy & 8 & $\circ$ & $\circ$ & 0 & $\circ$ & 0 & $\stackrel{\mathbb{D}}{\Omega}$ \\
\hline Totals & 50 & $\mathbf{I}$ & Io & $\circ$ & $\mathbf{I}$ & $\circ$ & 3 \\
\hline
\end{tabular}

These results extend over a period of six to twelve months after operation.

was one death occurring 24 hours after operation. The patient complained of a sudden pain in the chest, sat up and died within a few seconds. There was no evidence of a pulmonary embolus and unfortunately an autopsy was refused.

\section{Conclusions}

Resection treatment, which closely approximates a cure for the disease, is favourably regarded today by thoracic surgeons as it offers a means of actual removal of permanently damaged lung tissue.

A short period only of hospitalization is neces- sary, which is of great importance in these days of bed shortage.

The patient welcomes this form of treatmenti which, to him, shows a reasonable line of approach, and psychologically he benefits as he no longerconsiders himself ' a tuberculous outcast.?

This paper deals with the indications for resection in pulmonary tuberculosis, the technique $\vec{z}$ of resection and the complications that maye arise.

The post-operative physiotherapy, the $\cos -\overrightarrow{0}$ valescence and the follow-up of the patient briefly outlined.

\section{ANNOTATION}

\section{Glucose Tolerance Tests}

A reduction of the body's power to dispose of ingested carbohydrate results in its failure to maintain the blood sugar concentration constantly within normal limits. In order to reveal such a deficiency, sugar metabolism as a whole may be strained by the imposition of a glucose load upon it, abnormally high blood sugar levels afterwards showing that glucose tolerance is reduced. Glucose tolerance tests are designed with this object, and in the past 30 to 40 years the methods and results, in normal and abnormal states, have been standardized by various investigators.

The most commonly employed test involves the estimation of the blood sugar at half-hourly, and the urinary sugar at hourly intervals for two hours after the administration of $50 \mathrm{gm}$. glucose by mouth. Criteria of normality have been established for this test by its performance upon series of healthy young adults. The blood sugar should be below $120 \mathrm{mg}$. per $100 \mathrm{ml}$. with the subject fasting, and findings above this level indicate reduced tolerance (Mosenthal, 1947; Moyer and Womack, I950). It is agreed by most that the venous blood sugar two hours after taking the glucose should noto normally exceed $120 \mathrm{mg}$. per $100 \mathrm{ml}$. (Hale-White and Payne, 1926; Langner and Dewers, 1942). Many consider that this particular blood sugar N level is the only accurate criterion of sugar toler-n ance. The blood sugar level attained at the inter-N mediate times during the test is of debatableo significance. Joslin (1946) considered that non-o fasting sugar concentrations above $170 \mathrm{mg}$. per rooळ $\mathrm{ml}$. in venous blood and $200 \mathrm{mg}$. per $100 \mathrm{ml}$. in capillary blood indicated diabetes mellitus, andō̄ hence reduced tolerance if accompanied by glyco- $-\frac{\mathbb{Q}}{\mathbb{Q}}$ sturia. Spence (1920), MacLean and de Wesseloẘ 